\title{
Características clínicas, demográficas y epidemiológicas de una cohorte de pacientes con sindrome de Takotsubo entre 2011 y 2016 en Medellín, Colombia
}

\author{
Sebastián Naranjo-Restrepo* \\ Ana G. Múnera-Echeverri** \\ María C. Gaviria-Aguilar*** \\ Daniela Gutiérrez-Prieto**** \\ Elsa María Vásquez-Trespalacios***** \\ Mauricio Duque-Ramírez ${ }^{* * * * *}$
}

*Cardiólogo. CES Cardiología, Universidad CES. Medellín. Antioquia. Colombia.

**Cardióloga, ecocardiografista. Hospital General de Medellín. Universidad CES, Medellín. Antioquia. Colombia.

***Residente de Medicina Interna. Universidad CES. Medellín. Antioquia. Colombia.

****Médica general. Universidad CES. Medellín. Antioquia. Colombia

*****Epidemióloga. Universidad CES. Medellín. Antioquia. Colombia.

******Cardiólogo, electrofisiólogo. Universidad CES, CES Cardiología. Medellín. Antioquia. Colombia.

Correspondencia: Dr. Mauricio Duque. Dirección: Calle 34 \# 43-66, Centro comercial San Diego, Torre Norte (Falabella), piso 11. CES Cardiología. Medellín, Colombia. Teléfono: (+57) (4) 44473 78. Correo electrónico: mauricioduquemd@gmail.com

Resumen

Introducción: El síndrome de Takotsubo es una disfunción ventricular aguda de carácter temporal, usualmente reversible, secundaria a eventos estresantes que comprometen el eje cerebro-corazón. Objetivo: describir las características clínicas, epidemiológicas y desenlaces de una cohorte de pacientes con síndrome de Takotsubo en Medellín-Colombia. Materiales y métodos: Estudio observacional, descriptivo, ambispectivo, realizado en una cohorte de pacientes. Se revisaron las historias clínicas de pacientes que ingresaron al servicio de urgencias de una institución de tercer nivel de complejidad de la ciudad de Medellín entre enero de 2011 y diciembre de 2016 con diagnóstico de síndrome de Takotsubo. Se diseñó un cuestionario para recolectar variables demográficas, clínicas, de laboratorio e imagenológicas. Se obtuvieron medidas de frecuencia y análisis estadístico con el programa SPSS versión 21. Resultados: El 66,7\% de los pacientes fueron mujeres, la mortalidad fue del $11,1 \%$ y la recurrencia del 5,6\%. La mayoría de los casos tuvieron un desencadenante conocido (94,5\%), siendo la infección el más frecuente (44,4\%). El $77,8 \%$ de los pacientes presentó disnea al ingreso y el $27,8 \%$ requirió uso de algún vasopresor. El promedio de la fracción de eyección del ventrículo izquierdo inicial fue del 34.17+7.8 con evidencia de mejoría en el control, promedio luego del control= 54,81+10.2 Discusión y conclusiones: El síndrome de Takotsubo es una condición infrecuente, las mujeres son la población más afectada y la mayoría de los casos obedece a un factor desencadenante. La mortalidad reportada está dentro de lo esperado y se evidenció un buen pronóstico a mediano plazo. MÉD.UIS. 2020;34(1):27-33.

Palabras clave: Cardiomiopatía. Síndrome de Tako-tsubo. Disfunción. Ventricular. Síndrome del corazón roto.

\section{Clinical, demographic and epidemiological characteristics of a cohort of patients with Takotsubo's syndrome from 2011 to 2016 in Medellin, Colombia}

Abstract

Introduction: Takotsubo syndrome is an acute, usually reversible, temporal ventricular dysfunction secondary to stressful events that compromise the brain-heart axis. The study's objective is to describe the clinical, epidemiological characteristics and outcomes of a 
cohort of patients with Takotsubo syndrome in Medellin-Colombia. Methods: Observational, descriptive, ambispective study performed in a cohort of patients. We reviewed the medical records of patients who entered the emergency department of a third-level complex institution of Medellin between January 2011 and December 2016 with Takotsubo syndrome diagnosis. A questionnaire was designed to collect demographic, clinical, laboratory, and imaging variables. Frequency measures and statistical analyses were obtained with the SPSS version 21 program. Results: $66.7 \%$ of the patients were women, mortality was $11.1 \%$, and recurrence was $5.6 \%$. The majority of the cases had a known trigger (94.5\%), being the infection the most frequent (44.4\%), $77.8 \%$ of the patients presented dyspnea on admission, and $27.8 \%$ required the use of vasopressor. The mean baseline left ventricular ejection fraction was $34.17+7.8$ with evidence of improvement in control, average after control $=54.81+10.2$. Discussion and conclusions: Takotsubo syndrome is an uncommon condition. Women are the most affected population and most cases are due to a secondary cause. The reported mortality is within expectations, and a good medium-term cardiac prognosis was evidenced. MÉD.UIS. 2020;34(1):27-33.

Keywords: Cardiomyopathy. Tako-tsubo syndrome. Ventricular. Dysfunction. Broken Heart syndrome.

¿Cómo citar este artículo?: Naranjo-Restrepo S, Múnera-Echeverri AG, Gaviria-Aguilar MC, Gutiérrez-Prieto D, Vásquez-Trespalacios EM, Duque-Ramírez M. Características clínicas, demográficas y epidemiológicas de una cohorte de pacientes con síndrome de Takotsubo entre 2011 y 2016 en Medellín, Colombia. MÉD.UIS. 2020;33(2):27-33. doi: 10.18273/revmed.v34n1-2021003

\section{Introducción}

Elsíndromede Takotsubo(ST), también conocido como síndrome de balonamiento apical transitorio, síndrome del corazón roto o miocardiopatía por estrés, es una falla cardíaca aguda caracterizada por disfunción temporal ventricular, usualmente reversible y en la cual hay ausencia de lesión coronaria oclusiva que la explique'.

Se han descrito múltiples mecanismos fisiopatológicos causantes, como la toxicidad miocárdica por el aumento en catecolaminas circulantes, disfunción del sistema nervioso autónomo con hiperactividad del simpático, reacción inflamatoria con elevación de interleuquinas séricas, isquemia miocárdica con espasmos de las coronarias y alteraciones microvasculares, factores hormonales, predisposición genética, entre otros, desencadenados por un evento de estrés físico o emocional intenso ${ }^{2-3}$.

Es más frecuente en mujeres con una relación mujer: hombre de 9:1, principalmente postmenopáusicas. Sin embargo, se reportan más complicaciones intrahospitalarias como arritmias ventriculares, necesidad de soporte ventilatorio y paro cardiaco en la población masculina, otros factores relacionados con peor pronóstico son la presencia de factor desencadenante, cuadro neurológico agudo o enfermedad psiquiátrica, niveles de troponina elevados y disminución de la fracción de eyección $(<45 \%)^{4}$. En mujeres son más frecuentes los desencadenantes emocionales, mientras que en los hombres es más prevalente el estrés físico (choque, sangrado intracerebral, entre otros) como factor desencadenantes.

Como cardiopatía fue descrito por primera vez en 1990 por Sato et al. (Japón) como una forma de aturdimiento miocárdico agudo catecolaminérgico, sin una lesión coronaria oclusiva que explique el patrón de disfunción temporal observado en el ventrículo izquierdo ${ }^{6}$. Clínicamente semeja el síndrome coronario agudo, pero con reversibilidad de los hallazgos clínicos e imagenológicos en la mayoría de casos en un lapso corto de tiempo ${ }^{7-8}$.

Se ha descrito el dolor torácico como el síntoma de presentación más común (76\%), también se pueden presentar con disnea, síncope, síntomas vagales, palpitaciones, síntomas de falla cardiaca aguda y muerte súbita, o puede presentarse de forma asintomática9.

Aunque se considera una patología con pronóstico benigno puede traer consecuencias y secuelas graves, ocasionando una mortalidad intrahospitalaria del 2 al $5 \%{ }^{10}$ y una tasa de recurrencia entre el 5 al 22\%. Dentro de sus principales complicaciones se encuentran la falla cardíaca aguda, la obstrucción del tracto de salida del ventrículo izquierdo, la regurgitación mitral, choque cardiogénico, arritmias (como taquicardia sinusal, complejos atriales y ventriculares prematuros, taquicardia supraventricular paroxística, fibrilación atrial, taquicardia ventricular, fibrilación ventricular, bloqueo de rama izquierda y derecha así como bradiarritmias, asistolia y actividad eléctrica sin pulso), formación de trombos intracavitarios con fenómenos embólicos y la ruptura del ventrículo izquierdo ${ }^{11}$. 
Enero - abril

Es una enfermedad infrecuente, con pocos datos epidemiológicos reportados en Latinoamérica. Además poco diagnosticada por el desconocimiento de la misma y por las similitudes en sus manifestaciones clínicas con el síndrome coronario agudo; condición que puede descartarse mediante la angiografía coronaria diagnóstica temprana ${ }^{12}$.

A nivel mundial, se estima que las tasas de recurrencia y mortalidad a 5 años son entre el $5-22 \%$ y entre el $3-17 \%$, respectivamente ${ }^{1,10,13-14}$. La mayoría de datos provienen de países asiáticos, europeos y de Norte América, puesto que los datos existentes en países latinoamericanos acerca de presentación clínica, seguimiento y desenlaces a corto y mediano plazo del ST se limitan solo a reportes de $\operatorname{casos}^{15-16}$. En vista de esta falta de información, que termina dificultando el diagnóstico oportuno y de la relevancia clínica de las complicaciones, recurrencia y mortalidad de la misma, se decidió estudiar las características de los pacientes con ST en una muestra de una ciudad de Latinoamérica.

El objetivo de este estudio es describir las características clínicas, epidemiológicas y los desenlaces de una cohorte de pacientes con ST entre 2011 y 2016 en la ciudad de Medellín, Colombia.

\section{Materiales y métodos}

Este es un estudio observacional de tipo descriptivo, ambispectivo. Los criterios de inclusión son pacientes mayores de 18 años que ingresaron al servicio de urgencias de un hospital de tercer nivel de complejidad de la ciudad de Medellín, Colombia, en el período comprendido entre enero de 2011 y diciembre de 2016, con cuadro clínico de falla cardiaca aguda y ecocardiografía inicial compatible con ST y con control ecocardiográfico donde se evidenciara recuperación de la función ventricular como principal parámetro, entre otros. Se realizó un censo, analizando todas las historias clínicas que cumplieran con los criterios de inclusión. No se consideraron criterios de exclusión por lo selecto y específico de la población que se estaba estudiando. Los datos de las bases de datos fueron extraídos durante los años 2015 y 2016. Se obtuvieron variables demográficas, antecedentes personales, manifestaciones clínicas, signos vitales, probables desencadenantes como estrés físico y/o emocional, biomarcadores cardíacos, imágenes (angiografía coronaria, hallazgos ecocardiográficos de ingreso y control), días de estancia hospitalaria, tratamiento recibido, desenlaces (muerte, recuperación o recurrencia). Posterior al alta se hizo seguimiento telefónico a todos los pacientes para conocer la supervivencia en un lapso mínimo de 6 meses, así como la frecuencia de recurrencia.

El análisis estadístico se realizó con el programa SPSS versión 21, para los datos descriptivos se obtuvieron promedios y medianas con sus respectivas medidas de dispersión. Los cambios ecográficos iniciales y de control se analizaron mediante pruebas de comparación de promedios para muestras repetidas (Comparación de promedios con la prueba $U$ de Mann-Whitney).

\section{Resultados}

Se recolectaron 18 pacientes, cuyo promedio y desviación estándar de la edad fue de $59.5 \pm 16.02$ años, el $66.7 \%$ fueron mujeres. Entre los antecedentes personales más comunes destacan hipertensión arterial (50,0\%), tabaquismo (44,4\%), trastornos psiquiátricos (27,8\%) y diabetes mellitus (22,2\%), en la tabla 1 se detallan las características demográficas, los antecedentes personales de los pacientes y el valor de troponinas iniciales y de control, en este punto es importante aclarar que el valor de referencia del laboratorio es $<0,04$ y que las troponinas finales solo se midieron en 5 pacientes según criterio médico.

Tabla 1. Características demográficas y antecedentes personales

\begin{tabular}{|l|c|}
\hline Variable & (n=18) \\
\hline Edad Promedio $\pm \mathrm{SD}^{*}$ (años) & $59,5 \pm 16.02$ \\
\hline Mujeres, $\mathrm{n}(\%)$ & $12(66,7)$ \\
\hline Troponina Inicial & $1,59 \pm 1,67^{*}$ \\
\hline Troponina Final & $5,8 \pm 9,3^{*}$ \\
\hline Antecedentes personales & $\mathbf{n}(\%)$ \\
\hline Hipertensión arterial & $9(50,0)$ \\
\hline Tabaquismo & $8(44,4)$ \\
\hline Trastornos psiquiátricos ${ }^{\dagger}$ & $5(27,8)$ \\
\hline Diabetes mellitus & $4(22,2)$ \\
\hline Enfermedad coronaria & $2(11,1)$ \\
\hline Obesidad & $1(5,6)$ \\
\hline Reemplazo de válvula biológica & $1(5,6)$ \\
\hline Consumo de otras sustancias psicoactivas & $1(5,6)$ \\
\hline VIH & $1(5,6)$ \\
\hline Enfermedad cerebrovascular & $1(5,6)$ \\
\hline
\end{tabular}

* Desviación estándar.

† Trastornos psiquiátricos: Depresión (2 pacientes), trastorno afectivo bipolar (1 paciente), trastorno de ansiedad (1 paciente), duelo no resuelto (1 paciente).

Fuente: autores 
Los síntomas más frecuentes fueron disnea, dolor precordial y muerte súbita cuya frecuencia de presentación fue del $77,8 \%$, 50,0\% y $11,1 \%$, respectivamente. En el $94,5 \%$ de los casos se logró documentar el factor desencadenante, siendo el mas importante el estrés físico (77,8\%), y de este, los mas relevantes fueron los procesos infecciosos 44.4\% (colecistitis, infección urinaria, pielonefritis, infección respiratoria, diarrea y colecciones pélvicas). El estrés emocional estuvo presente en el $16,7 \%$ de los casos y en $5.6 \%$ de los pacientes no se encontró el factor desencadenante del síndrome (Takotsubo primario) (ver tabla 2). Cabe resaltar que en los desencadenantes mencionados, las variables tanto de estrés físico como emocional no son excluyentes y por lo tanto, el porcentaje puede superar el $100 \%$.

Tabla 2. Síntomas y desencadenantes.

\begin{tabular}{|l|l|}
\hline Síntomas & $\mathbf{n}(\%)$ \\
\hline Disnea & $14(77,8)$ \\
\hline Dolor precordial & $9(50,0)$ \\
\hline Muerte súbita & $2(11,1)$ \\
\hline Mareo & $1(5,6)$ \\
\hline Desencadenantes & $\mathbf{n}(\%)$ \\
\hline Estrés físico & $14(77,8)$ \\
\hline Infección & $8(44,4)$ \\
\hline Otros* & $5(27,8)$ \\
\hline Sepsis & $4(22,2)$ \\
\hline Postquirúrgico ${ }^{\ddagger}$ & $3(16,7)$ \\
\hline Estrés emocional & $3(16,7)$ \\
\hline Trastorno de ansiedad & $2(11,1)$ \\
\hline Trastorno depresivo & $1(5,6)$ \\
\hline Malas noticias & $1(5,6)$ \\
\hline Sin causa aparente & $1(5,6)$ \\
\hline & Otros: Adrenalin \\
\hline
\end{tabular}

* Otros: Adrenalina intravenosa (1 paciente), crisis asmática

(1 paciente), intoxicación por litio (1 paciente), intoxicación feniletilamina (1 paciente) y anemia hemolítica (1 paciente).

‡ Postquirúrgico: toracoscopia, herniorrafia, laparotomía exploratoria.

Fuente: autores

Los medicamentos más frecuentemente utilizados para el manejo del ST de forma intrahospitalaria fueron los beta-bloqueadores en el $83,3 \%$ de los casos, seguido de diuréticos $66,7 \%$ e IECA/ARAll, que sumados fueron suministrados en el $83,4 \%$ de los casos. Cabe anotar que el $27,8 \%$ de los pacientes requirió algún tipo de vasopresor durante la estancia hospitalaria. Los demás medicamentos se enuncian en la tabla 3.

Tabla 3. Medicamentos utilizados para el manejo intrahospitalario.

\begin{tabular}{|l|l|}
\hline Medicamentos & $\mathbf{n}(\%)$ \\
\hline Beta-bloqueador & $15(83,3)$ \\
\hline Diurético & $12(66,7)$ \\
\hline IECA $\ddagger$ & $11(61,1)$ \\
\hline Calcioantagonistas & $6(33,3)$ \\
\hline Vasopresores & $5(27,8)$ \\
\hline ARA-II $\boldsymbol{1}$ & $4(22,2)$ \\
\hline
\end{tabular}

¥ Inhibidores de la enzima convertidora de angiotensina.

II Antagonistas de los receptores de angiotensina II.

Fuente: autores

Los hallazgos ecocardiográficos al momento del ingreso, comparados con el control realizado a los 29 días en promedio del evento, se muestran en la tabla 4. Se usó como prueba estadística la comparación de promedios con la prueba U de Mann-Whitney.

En todos los casos se observó un aumento de la fracción de eyección del ventrículo izquierdo (FEVI) en promedio de $20.64 \%$, con una mejoría tanto en el volumen de la aurícula izquierda (AI) así como de la presión sistólica de la arteria pulmonar (PSAP).

Los datos de coronariografía estuvieron disponibles en 14 de 18 pacientes $(77,8 \%)$. Se documentó en 2 de ellos enfermedad coronaria que angiográficamente no explicaba los cambios observados en el ecocardiograma transtorácico (TT). La mortalidad global de la cohorte fue del 11.1\%: 2 de 18 pacientes murieron a los 4 y 11 meses posteriores al evento, al parecer por causas no relacionadas con el ST, sin embargo, no se realizó análisis de mortalidad atribuible. No hubo ningún caso de muerte intrahospitalaria. Solo el 5,6\% de los pacientes presentó recurrencia del ST en el año siguiente (ver tabla 5).

\section{Discusión}

El ST fue descrito por primera vez en 1990 y en el 2006 fue clasificado como una cardiomiopatía adquirida primaria ("Tako-Tsubo", cardiomiopatía por estrés), la cual es mediada por fenómenos vasculares, miocárdicos o mixtos que ocurren posterior a un evento de estrés emocional o físico ${ }^{1,3,7,17-18}$. Dicha enfermedad simula clínicamente un síndrome coronario agudo (SCA $)^{8}$, sin embargo su incidencia es baja, únicamente entre el 1 al $2 \%$ de los pacientes con sospecha de SCA terminan con diagnóstico de 


\section{Enero - abril}

ST después de que se excluyen lesiones coronarias oclusivas ${ }^{1,3,7,17-19}$.

La mayoría de los datos epidemiológicos del ST provienen de Norteamérica, países asiáticos, europeos y poblaciones caucásicas y negras. En Latinoamérica solo se dispone de reportes de caso y revisiones de tema ${ }^{15-20,21-22}$. Es por esto que en la discusión no se compara con Artículos Originales con poblaciones demográficamente similares a la presente en este estudio.

Similar a lo reportado en la literatura, la mayoría de pacientes fueron mujeres, sin embargo la relación mujer:hombre fue 3:1, inferior a lo descrito por
Sharkey et al. $^{23}$ y otros autores ${ }^{1,18}$ que estiman una relación 9:1. En este estudio el promedio de edad fue de 59,3+19, acorde a lo reportado por otras series en las que el ST típicamente afecta a mujeres mayores de 50 años post-menopáusicas ${ }^{5,10}$. En el $94.5 \%$ de los casos en que se logró establecer el factor desencadenante del ST, el estrés físico fue la principal causa de éste, tanto en hombres como en mujeres (77\% de los casos) ; sin embargo, en los casos en los que el estrés emocional fue el desencadenante solo las mujeres se vieron afectadas (16,7\% del total de los pacientes). Además existen otros trabajos como el de Sharkey et al, en el que se logró establecer el factor desencadenante en el $89 \%$ de los pacientes, de estos $47 \%$ fueron causas emocionales y $46 \%$ estrés físico ${ }^{23}$.

Tabla 4. Cambios ecocardiográficos iniciales y de control

\begin{tabular}{|l|l|l|l|l|}
\hline Variable & Valores de referencia & Ecocardiograma TT* al ingreso. Promedio & $\begin{array}{l}\text { Ecocardiograma TT* control. } \\
\text { Promedio }\end{array}$ & Valor de $\mathbf{P}$ \\
\hline FEVI $^{+}(\%)$ & $52-72 \%$ & $34,17(17-48)^{* *}$ & $54,81(33-70)^{* *}$ & $<0,0001$ \\
\hline${\text { Volumen } \mathrm{Al}^{\ddagger}(\mathrm{ml} / \mathrm{m} 2)}^{*}$ & $16-34 \mathrm{ml} / \mathrm{m} 2$ & $32,94(17-60)^{* *}$ & $27,5(11-57)^{* *}$ & $<0,001$ \\
\hline PSAP" $\left.^{*} \mathrm{mmHg}\right)$ & $<35 \mathrm{~mm} \mathrm{Hg}$ & $48,61(31-63)^{* *}$ & $35,27(25-47)^{* *}$ & $<0,001$ \\
\hline
\end{tabular}

*TT: Transtorácico.

† FEVI: Fracción de eyección del ventrículo izquierdo.

‡ Al: Aurícula Izquierda.

ๆ PSAP: Presión sistólica de la arteria pulmonar.

** Intervalo

Fuente: autores

Tabla 5. Desenlaces del ST

\begin{tabular}{|l|l|}
\hline Variable & $\mathbf{( n = 1 8 )}$ \\
\hline Promedio días de hospitalización & $18(3-91)^{*}$ \\
\hline Promedio días de seguimiento & $739(180-2010)^{*}$ \\
\hline
\end{tabular}

* (Intervalo: Días mínimos - Días máximos)

Fuente: autores

En el $77 \%$ de pacientes se realizó el diagnóstico de ST por clínica, recuperación de la FEVI en ecocardiograma TT de control y coronariografía sin evidencia de lesiones coronarias obstructivas significativas, mientras que en el porcentaje restante el diagnóstico se basó en la evolución clínica y la recuperación de la FEVI por ecocardiografía, sin tener documentación de coronariografía. En otros centros se utilizan diferentes métodos diagnósticos como medicina nuclear, resonancia magnética, angiotomografía, entre otros ${ }^{11}$.

En general, el tratamiento en la fase aguda es de soporte y manejo de las complicaciones. En casos leves, se podría considerar el uso de inhibidores de la enzima convertidora de angiotensina o antagonistas del receptor de angiotensina II y beta-bloqueadores ${ }^{2}$. En caso de falla cardiaca aguda con edema pulmonar, se indican los diuréticos ${ }^{14} \mathrm{y}$ en pacientes con choque cardiogénico, recientemente se ha sugerido que el levosimendán podría ser usado como una opción más segura"1

El 27,8\% de los pacientes requirió uso de vasopresor en algún momento de la hospitalización para el manejo de la falla cardiaca aguda, este dato es similar a lo descrito por Nascimento et al. en una cohorte de 59 pacientes recolectados en el hospital Mount Sinai Heart Institute donde el 20\% requirieron vasopresor ${ }^{22}$.

En este estudio se reportó una mortalidad del $11,1 \%$, cifra que está dentro de lo reportado a nivel mundial $(3-17 \%)^{1,22}$, no se presentaron muertes de forma intrahospitalaria que según otras series pueden oscilar alrededor del 2 al 5\%5,10,13. De acuerdo con otros reportes ${ }^{16}$, la mejoría ecocardiográfica es temprana, suele ocurrir en las primeras 4 semanas; 
en ésta cohorte se evidencia una mejoría tanto de la FEVI como de la PSAP y del volumen de la AI en el control ecocardiográfico.

Aunque considerada una entidad benigna por su potencial reversibilidad en el tiempo y pronóstico favorable, el ST puede tener complicaciones potencialmente fatales como falla cardíaca aguda (45\%), obstrucción del tracto de salida del ventrículo izquierdo (25\%), shock cardiogénico (20\%) y arritmias (24\%), incluso hasta ruptura del ventrículo izquierdo ${ }^{1,10}$. En este trabajo, durante la hospitalización y seguimiento, no se presentaron otras complicaciones mayores como: trombosis, accidente cerebrovascular, ruptura ventricular izquierda, obstrucción del tracto de salida, regurgitación mitral severa, arritmias o choque cardiogénico.

A continuación, se describen algunas de las limitaciones del estudio: esta investigación es un estudio retrospectivo que fue desarrollada en un solo centro. El criterio diagnóstico se fundamentó en los hallazgos ecocardiográficos, angiográficos y clínicos, cabe aclarar que en el $22,2 \%$ de los pacientes no se realizó coronariografía, el diagnóstico fue basado en la evolución clínica y la recuperación de la FEVI por ecocardiografía. Además, no se consideraron criterios de exclusión por lo selecto y específico de la población que se estaba estudiando.

\section{Conclusiones}

En el presente estudio, la edad promedio de presentación fue de 59,3+19, la mayoría de los pacientes afectados fueron mujeres con causas secundarias, y a diferencia de otras series, la relación mujer: hombre fue menor, cabe resaltar que los síntomas mas frecuentemente encontrados fueron disnea, dolor precordial y muerte súbita, difiriendo con los más comunes reportados en la literatura, en donde el síncope cobra un papel importante y la muerte súbita es infrecuente. A pesar de que puede recurrir y llevar a la muerte, estos dos desenlaces fueron infrecuentes en esta cohorte y se comportaron dentro de lo esperado. El pronóstico cardíaco a mediano plazo es bueno, evidenciado por la recuperación de la FEVI en la ecocardiografía TT. El 1-2\% de las sospechas de SCA, terminan siendo en realidad un Síndrome de Takotsubo, que puede llevar a complicaciones agudas graves como falla cardíaca aguda, obstrucción del tracto de salida del ventrículo izquierdo, choque cardiogénico, arritmias, entre otras; por lo tanto, el mejor entendimiento de esta patología en una muestra latinoamericana permitirá mejorar la oportunidad diagnóstica y, por ende, un adecuado seguimiento y tratamiento.

\section{Fuente de financiación}

Ninguna. La presente investigación no ha recibido ninguna beca específica de agencias de los sectores público, comercial, o sin ánimo de lucro.

\section{Conflictos de Intereses}

Todos los autores declaran que no existe ningún conflicto de interés en el presente artículo.

\section{Referencias bibliográficas}

1. Lyon AR, Bossone E, Schneider B, Sechtem U, Citro R, Underwood SR, et al. Current state of knowledge on Takotsubo syndrome: a Position Statement from the Taskforce on Takotsubo Syndrome of the Heart Failure Association of the European Society of Cardiology. Eur J Heart Fail. 2016;18(1):8-27.

2. Y-Hassan S, Tornvall P. Epidemiology, pathogenesis, and management of takotsubo syndrome. Clin Auton Res. 2018;28(1):53-65.

3. Allen D, Parmar G, Ravandi A, Hussain F, Kass M. Happiness can break your heart: a rare case of takotsubo cardiomyopathy after good news. Can J Cardiol. 2015;31(2):228.e1-2.

4. Dias A, Núñez Gil IJ, Santoro F, Madias JE, Pelliccia F, Brunetti ND, et al. Takotsubo syndrome: State-of-the-art review by an expert panel - Part 1. Cardiovasc Revasc Med. 2019;20(1):70-79.

5. Brinjikji W, El-Sayed AM, Salka S. In-hospital mortality among patients with takotsubo cardiomyopathy: a study of the National Inpatient Sample 2008 to 2009. Am Heart J. 2012;164(2):215-21.

6. Sato H, Tateishi H, Uchida T. Takotsubo-type cardiomyopathy due to multivessel spasm. In: Clinical aspect of myocardial injury; from ischemia to heart failure. Kagakuhyouronsya Co. 1990;56-64.

7. Lyon AR, Rees PSC, Prasad S, Poole-Wilson PA, Harding SE. Stress (Takotsubo) cardiomyopathy--a novel pathophysiological hypothesis to explain catecholamine-induced acute myocardial stunning. Nat Clin Pract Cardiovasc Med. 2008;5(1):22-9.

8. Sealove BA, Tiyyagura S, Fuster V. Takotsubo cardiomyopathy. J Gen Intern Med. 2008;23(11):1904-8.

9. Moscatelli S, Montecucco F, Carbone F, Valbusa A, Massobrio L, Porto I, et al. An Emerging Cardiovascular Disease: Takotsubo Syndrome. BioMed Res Int. 2019 Oct 30;2019:6571045. doi: 10.1155/2019/6571045. PMID: 31781633; PMCID: PMC6875025.

10. Citro R, Rigo F, D'Andrea A, Ciampi Q, Parodi G, Provenza G, et al. Echocardiographic correlates of acute heart failure, cardiogenic shock, and in-hospital mortality in tako-tsubo cardiomyopathy. JACC Cardiovasc Imaging. 2014;7(2):119-29.

11. Dias A, Núñez Gil IJ, Santoro F, Madias JE, Pelliccia F, Brunetti ND, et al. Takotsubo syndrome: State-of-the-art review by an expert panel - Part 2. Cardiovasc Revasc Med. 2019; 20(2):153-66.

12. Ali M, Rigopoulos AG, Ali K, Ikonomidis I, Makavos G, Matiakis $\mathrm{M}$, et al. Advancements in the diagnostic workup, prognostic evaluation, and treatment of takotsubo syndrome. Heart Fail Rev. 2020;25(5):757-771. PubMed PMID: 31372789.

13. Singh K, Carson K, Usmani Z, Sawhney G, Shah R, Horowitz J. Systematic review and meta-analysis of incidence and correlates of recurrence of takotsubo cardiomyopathy. Int J Cardiol. 2014;174(3):696-701. PubMed PMID: 24809923.

14. Kurowski V, Kaiser A, Von Hof K, Killermann DP, Mayer B, Hartmann F, et al. Apical and midventricular transient left 
Características clínicas, demográficas y epidemiológicas de una cohorte de pacientes con síndrome de Takotsubo entre 2011 y 2016 en Medellín, Colombia

ventricular dysfunction syndrome (Tako-Tsubo cardiomyopathy): Frequency, mechanisms, and prognosis. Chest. 2007;132(3):80916. PubMed PMID: 17573507.

15. Aguirre DM, Mejía DT, Atehortua DFL, Florez JS, Vasquez EVO. Enfermedad del corazón roto o síndrome de Tako Tsubo. MÉD. UIS. 2011;24(1):123-7.

16. Akashi YJ, Nakazawa K, Sakakibara M, Miyake F, Koike H, Sasaka $\mathrm{K}$. The clinical features of takotsubo cardiomyopathy. QJM. 2003;96(8):563-73. PubMed PMID: 12897341.

17. Yoshikawa T. Takotsubo cardiomyopathy, a new concept of cardiomyopathy: Clinical features and pathophysiology. Int J Cardiol. 2015;182:297-303. PubMed PMID: 25585367.

18. Templin C, Ghadri JR, Diekmann J, Napp LC, Bataiosu DR, Jaguszewski M, et al. Clinical Features and Outcomes of Takotsubo (Stress) Cardiomyopathy. N Engl J Med. 2015;373(10):929-38. PubMed PMID: 26332547.
19. Boland TA, Lee VH, Bleck TP. Stress-Induced Cardiomyopathy. Crit Care Med. 2015;43(3):686-93. PubMed PMID: 25565459.

20. Sénior JM, Tamayo-Artunduaga N, Fernández-Cadavid A, Rodríguez-Dimuro A. Cardiomiopatía de Takotsubo. Iatreia. 2015;28(2):202-6.

21. Lopera LM, Restrepo CE, Lopera-Rojas O, Mendoza JC. Rev Colomb Anestesiol. 2012;40(3):240-4.

22. Nascimento FO, Santana O, Perez-Caminero M, Benjo AM. The characteristics of stress cardiomyopathy in an ethnically heterogeneous population. Clinics (Sao Paulo). 2011;66(11):1895-9. PubMed PMID: 22086519; PubMed Central PMCID: PMC3203961.

23. Sharkey SW, Windenburg DC, Lesser JR, Maron MS, Hauser RG, Lesser JN, et al. Natural history and expansive clinical profile of stress (tako-tsubo) cardiomyopathy. J Am Coll Cardiol. 2010;55(4):333-41. PubMed PMID: 20117439. 